DFF-280/04/1997 (Florence)

JHU-TIPAC-97007 (Johns Hopkins)

April 1997

\title{
Tests of Basic Quantum Mechanics in Oscillation Experiments.
}

\author{
G. Domokos and S. Kovesi-Domokos \\ Dipartimento di Fisica, Universitá di Firenze \\ Florence, Italy \\ and \\ The Henry A. Rowland Department of Physics and Astronomy \\ The Johns Hopkins University] \\ Baltimore, MD 21218
}

\begin{abstract}
According to standard quantum theory, the time evolution operator of a quantum system is independent of the state of the system. One can, however, consider systems in which this is not the case: the evolution operator may depend on the density operator itself. The presence of such modifications of quantum theory can be tested in long baseline oscillation experiments.
\end{abstract}

While quantum theory works remarkably well and there is no evidence to date that its validity may be limited, there have been attempts at modifying its structure both in order to resolve some conceptual problems or in order to establish its limits of validity. In particular, modifications of quantum theory have been considered in order to resolve the problem of transition to classical theory (the problem of "decoherence"). Briefly, it has to be made sure that if two states are macroscopically different, then there is no definite phase relation between them and thus, their superposition is not a physically admissible state. (Sometimes this is dubbed as the "problem of Schrödinger's cat", see for instance D'Espagnat [1].) It is generally thought that decoherence is a consequence of some kind of an interaction with the environment in

\footnotetext{
${ }^{1}$ Permanent address. E-mail: SKD@HAAR.PHA.JHU.EDU
} 
some general sense. For instance, it was conjectured that gravitational interactions cause decoherence once a body is sufficiently massive [2]. However, it is also conceivable that decoherence is an intrinsic property of quantum theory itself: after a sufficiently long lapse of time, phase relations are lost even between microscopic states and, therefore, macroscopic ones as well ("spontaneous decoherence"). Clearly, this requires a modification of the formalism of quantum theory as we know it. Such modifications have been repeatedly advocated by a variety of authors; a reasonably complete and up-to-date account of the problem of decoherence is given in the book by Omnès [3].

In this note we consider one class of such possible modifications and suggest that one can place limits on departures from the standard version of quantum theory in experiments currently being carried out or being planned.

The standard form of the time evolution operator of a quantum system described by its density operator, $\rho$ is given by the expression 2 :

$$
i \frac{\partial \rho}{\partial t}=[H, \rho],
$$

$H$ being the Hamiltonian of the system. Equation (1) is linear in $\rho$. This fact has remarkable consequences; perhaps the most important one is that if at a time a system was in a pure state (i.e. $\left.\rho^{2}\left(t_{0}\right)=\rho\left(t_{0}\right)\right)$, then it will remain in a pure state at all times. (Conversely, a statistical mixture will not evolve spontaneously into a pure state either.)

It is conceivable, however that, due to the presence of some, hitherto undetected small non linearity in equation (1), the property we just described is only an approximate one: if left alone for a sufficiently long time, the system spontaneously evolves into a mixture or a mixture contracts to some pure state.

In order to explore this possibility, we add a non linear term to the right hand side of the evolution equation, (11) and from now on investigate the evolution of a density operator obeying the equation:

$$
i \frac{\partial \rho}{\partial t}=[H, \rho]-i \frac{a}{T}\left(f(\rho)-\frac{1}{N} \operatorname{Tr} f(\rho)\right)
$$

Here $T$ is some characteristic time scale. The constant $a$ serves the purpose of normalizing the time dependence of the solutions due to the presence of the term added to (11). The function $f(\rho)$ governs the deviation of the evolution from standard quantum mechanics. (Not having any firm guiding principle, we shall experiment with some simple functional forms.) Here and for the rest of this paper we restrict ourselves to state spaces of dimension $N$.

\footnotetext{
${ }^{2}$ Natural units are used: $\hbar=c=1$
} 
A generalization to infinite dimensional state spaces appears to be straight forward; however, we have not explored it in detail.

In writing down (2) we were guided by some physical prejudices. Three of those are worth noting.

- We want to maintain probability conservation, therefore the term added to the right hand side of (11) is traceless. As a consequence,

$$
\frac{\partial \operatorname{Tr} \rho}{\partial t}=0,
$$

as in standard quantum mechanics.

- We wrote down the evolution equation in such a manner that it contains a characteristic time scale governing the deviation from standard quantum mechanics. (Alternatively, we could have, e.g. contemplated a deformation of the Heisenberg algebra. However, deformations of Lie algebras as discussed in the literature, contain dimensionless parameters. We consider $T$ a constant of Nature; there are no dimensionless constants of Nature we know of.[1)

- The term added to the evolution equation is local in time. One could have given, e.g. some memory to the system by making the evolution equation depend on $\rho$ taken at some past moment or upon an integral of $\rho$, etc. However, if eventually, one wants to construct manifestly Lorentz invariant theories, such terms are hard or impossible to incorporate.

We note that the class of modifications considered here is, in a sense, $a$ minimal one: apart from the linearity of the evolution equation, no other essential property of quantum theory (e.g. the superposition principle) is affected.

By making the transformation,

$$
\rho(t)=\mathrm{e}^{-i H t} \rho_{1}(t) \mathrm{e}^{i H t},
$$

the time dependence due to the Hamiltonian is eliminated; the quantity $\rho_{1}$ obeys the equation:

$$
\frac{\partial \rho_{1}}{\partial t}+\frac{a}{T}\left(f\left(\rho_{1}\right)-\frac{1}{N} \operatorname{Tr} f\left(\rho_{1}\right)\right)=0 .
$$

\footnotetext{
${ }^{3}$ Often, the fine structure constant is considered a constant of Nature. It is not, however, because its magnitude depends on the energy scale at which the measurement is performed.
} 
We notice that "total disorder", i.e.

$$
\rho_{1}=\frac{1}{N}
$$

is a zero of $f-1 / N \operatorname{Tr} f$. Whether or not it is also an attractor, depends on the functional form of $f$. (One can linearize around (5) in order to determine this; however, the linearized version gives no information about the size of the basin of attraction.) Due to the vanishing of $f-1 / N \operatorname{Tr} f$, the density matrix in equation (5) is stationary.

In order to make further progress, we now consider some simple examples. The main technical simplification introduced is that we further restrict the dimensionality of the state space: we take $N=2$; in this way, we can take advantage of the properties of the algebra of quaternions (equivalently, of the Pauli matrices).

In two dimensions, a density matrix is of the form,

$$
\rho=\frac{1}{2}(1+\mathbf{s} \cdot \sigma)
$$

where $\sigma$ stand for the Pauli matrices and $\mathbf{s}^{2} \leq 1$. Clearly, since $\rho$ and $\rho_{1}$ are unitarily equivalent, $\rho_{1}$ can be written in the same form as (6). Consider now,

Example \# 1:

$$
f\left(\rho_{1}\right)=\rho_{1}^{2} .
$$

Equation (4) can be solved immediately. We have:

$$
\mathbf{s}_{1}(t)=\mathbf{s}_{1}(0) \exp \left(\frac{-a t}{2 T}\right)
$$

Clearly, only $a>0$ makes sense from the physical point of view, since $\mathbf{s}_{1}^{2} \leq 1$. We take $a=2$ in order to normalize the time dependence to $\exp -t / T$. (Evidently, such a choice of $a$ merely serves to normalize the time dependence in an appropriate fashion; it does not influence the physics in any way.)

\section{Example \# 2:}

$$
f\left(\rho_{1}\right)=\rho_{1}^{3} .
$$

Just like in the case of equation (7), the evolution equation (雨) can be solved in a closed form. The equation reads:

$$
\frac{\partial \mathbf{s}_{1}}{\partial t}=-\frac{a}{8 T} \mathbf{s}_{1}\left(3+\mathbf{s}_{1}^{2}\right)
$$


Clearly, the direction of $\mathbf{s}_{1}$ is constant and by taking the scalar product of equation (10) with $\mathbf{s}_{1}$, one obtains an equation for the magnitude of the polarization, fi viz.

$$
\frac{\partial \mathbf{s}_{1}^{2}}{\partial t}=\frac{-a}{4 T} \mathbf{s}_{1}^{2}\left(3+\mathbf{s}_{1}^{2}\right)
$$

The solution of eq. (11) is:

$$
\begin{aligned}
\mathbf{s}_{1}^{2}(t) & =\mathbf{s}_{1}^{2}(0) \mathrm{e}^{-3 a t / 4 T} \\
& \times\left[3+\mathbf{s}_{1}^{2}(0)\left(1-\mathrm{e}^{-3 a t / 4 T}\right)\right]^{-1}
\end{aligned}
$$

Clearly, only $a>0$ is physically acceptable and one can choose $a=4 / 3$ in order to standardize the time dependence. Both examples considered so far are such that for physically acceptable values of the parameter $a$, total disorder (equation (5)) is an attractor and after times of the order of $T$ the quantum system will find itself in the neighborhood of the attractor.

This need not be the case, however. One can construct examples with other attractors. It would be tempting to choose for $f$ something like $\rho(1-\rho)$ in analogy with the logistics equation; presumably, such a term would drive the quantum system towards a pure state. However, that expression has no traceless part in two dimensions. One does not want any dimensionality to be singled out, thus some more complicated functional form has to be tried.

\section{Example \# 3: Choose:}

$$
f(\rho)=\rho^{3}-\rho^{2}
$$

Clearly, $f(\rho)=0$ for a pure state. Equation (\#) can be solved in a closed form for this case too. As before, it is sufficient to give the time evolution of the magnitude of $\mathbf{s}$. One has:

$$
\mathbf{s}^{2}(t)=\frac{\mathbf{s}^{2}(0)}{\mathbf{s}^{2}(0)+\left(1-\mathbf{s}^{2}(0)\right) \mathrm{e}^{-a t / 4 T}} .
$$

In this case, both positive and negative values of $a$ lead to physically acceptable results; however, the qualitative behavior of the system depends crucially on the sign of $a$.

\footnotetext{
4 "Polarization" is used in a generalized sense. In general, it is just a measure of the deviation from total disorder.
} 
1. If $a>0$, a pure state $\left(\mathbf{s}^{2}=1\right)$ is an attractor: if initially $\mathbf{s}^{2}<1$, the system will move towards a pure state unless initially $\mathbf{s}=0$. Any point within the unit ball with the origin removed is within the basin of attraction of a pure state.

2. The situation is reversed for $a<0$ : any point in the interior of the unit ball $\left(\mathbf{s}^{2}<1\right)$ is in the basis of attraction of total disorder (eq. (5)) and the surface is an unstable fixed point.

This distinction is relevant from the point of view of experimental tests.

The possibility of testing for the presence of terms proportional to $1 / T$ in the evolution equation of a quantum system arises from the fact that present and planned long baseline neutrino oscillation experiments take place on length scales of the order of $d \simeq 10^{3} \mathrm{~km}$; hence, they should be sensitive to characteristic times, $d=T \simeq 10^{-3} \mathrm{~s}$. This is considerably larger than the time scales involved in typical terrestrial experiments. (For comparison, a typical atomic transition is characterized by times of the order of $1 \mathrm{eV}^{-1} \simeq 10^{-15} \mathrm{~s}$; the time associated with the $K_{S}-K_{L}$ mass difference is about $(\Delta m)^{-1} \simeq$ $2 \times 10^{-10} \mathrm{~s}$.)

In principle, the test is a very simple one. We noticed that, as a consequence of probability conservation, a "total disorder" characterized by eq. (5) is always a fixed point of equation (四), and, hence, of the complete density operator. From the examples considered, it is also likely that total disorder is an attractor unless matters are specially arranged, as in Example \#3. Therefore, a likely test for the presence of non linear terms in the evolution equation consists of a search for spontaneous depolarization as a function of time.

In order to make matters more quantitative, let us consider neutrino oscillations with a non linear term in the evolution equation discussed in Example \#1 above. Neutrinos are particularly advantageous from the point of view of testing for spontaneous decoherence, since their interaction with the environment is generally negligibly small. Thus, one may be able to distinguish between spontaneous and environmental [2, 4, 3] decoherence.

Consider therefore a Hamiltonian of a two flavor system, say $\left(\nu_{\mu}, \nu_{e}\right)$ [5], with a Hamiltonian in the diagonal basis given as:

$$
H=\frac{E_{1}+E_{2}}{2}+\frac{E_{1}-E_{2}}{2} \sigma_{3}
$$

and a mixing matrix,

$$
U=\mathrm{e}^{i \theta \sigma_{2}}
$$


At momenta much higher than the rest masses of the neutrinos, the energies are given by the expressions:

$$
E_{i} \approx p+\frac{m_{i}^{2}}{2 p} \quad(i=1,2)
$$

The density matrix in the flavor basis at production is given by:

$$
\rho(0)=\frac{1}{2}\left(1+s_{3}(0) \sigma_{3}\right)
$$

The value of $s_{3}(0)$ in eq. (18) equals \pm 1 , depending on whether $\nu_{\mu}$ or $\nu_{e}$ is produced. Using the preceding equations and eq. (8), one readily obtains the density matrix at time $t$ :

$$
\begin{aligned}
\rho(t) & =1 / 2+1 / 2 s_{3}(0) \mathrm{e}^{-t / T} \sigma_{3}\left(\cos ^{2} \phi+\sin ^{2} \phi \cos 4 \theta\right) \\
& +1 / 2 s_{3}(0) \mathrm{e}^{-t / T} \sin ^{2} \phi\left(-\sigma_{1}+\sigma_{2}\right)
\end{aligned}
$$

where

$$
\phi=\frac{\Delta m^{2} t}{4 p}
$$

(In the limit of $T \rightarrow \infty$, eq. (19), of course, reproduces the standard result.) Similar expressions hold whenever total disorder is an attractor, as in the second example discussed above: in all such cases, the signature for a departure from standard quantum mechanics is a damping of the polarization.

Example \# 3 deserves special attention: the evolution equation has a fixed point at $\mathbf{s}^{2}=1$. It is generally assumed that weak interactions produce neutrinos of a definite flavor, i.e. in a pure state. If the fixed point in Example \# 3 is a stable one, one has virtually no chance of observing a deviation from standard quantum theory in a neutrino oscillation experiment, even though the evolution equation of the density matrix contains non linear terms. Even if the fixed point is unstable, one needs environmental perturbations in order to drive the neutrino away from a pure state: the relevant Lyapunov exponent may be too small to make the presence of a non linear term in the evolution equation observable. The lesson to be learnt from this example is that even though there may be deviations from standard quantum theory present in the evolution equation of the density operator, circumstances may conspire to effectively hide that deviation from experimental scrutiny.

To summarize, long baseline neutrino oscillation experiments are likely to provide an environment for testing the validity of standard quantum theory, due to the unusually long distances involved in such experiments. From the examples considered here, it appears that, if non linear terms are present in 
the evolution equation for the density matrix, they are likely to lead to spontaneous decoherence. Nevertheless, some caution is needed: there may be situations in which the presence of deviations from standard quantum theory is hidden from observation in certain experiments. One also has to bear in mind that, in all the examples considered in this paper, spontaneous decoherence leads to total disorder. This is not necessarily the general situation, however. One can think of a number of non linear extensions of the evolution equation for the density operator in which off diagonal elements go to zero as time increases, but the density operator does not become a multiple of the unit operator.

Acknowledgement. This work was done during the authors' visit at the Dipartimento di Fisica, Universitá di Firenze. We thank Roberto Casalbuoni, Director of the Department, for the hospitality extended to us. We also thank Frigyes Károlyházy for several tutorial sessions on the problem of decoherence.

\section{References}

[1] . B. D'Espagnat, Conceptual Foundations of Quantum Mechanics, $2^{\text {nd }}$ edition. Benjamin, Reading, MA (1976); Ch. 3.

[2] F. Károlyházy, Nuovo Cim. 42, 390 (1966). Also, F. Károlyházy, in "Sixty Two Years of Uncertainty", ed. A.I. Miller. Plenum, New York 1990.

[3] R. Omnès, The Interpretation of Quantum Mechanics. Princeton University Press, Princeton, NJ. (1994); Ch. 7.

[4] See e.g. W.H. Zurek, Phys. Rev. D 26, 1862 (1982); also, Physics Today, 44, 36 (1991).

[5] C.W. Kim and A. Pevsner, Neutrinos in Physics and Astrophysics, Harwood Academic Publishers, Chur (1993); Ch. 7. 